\title{
Effective high intensity focused ultrasound treatment in recurrent aggressive breast fibromatosis: a case report
}

This article was published in the following Dove Press journal: OncoTargets and Therapy

\author{
Jing Zhao' \\ Qiyuan Wang ${ }^{2}$ \\ Xiuzhen $\mathrm{Li}^{3}$ \\ Xiaoye $\mathrm{Hu}^{4}$ \\ Hong Shen' \\ 'Department of Medical Oncology, \\ The Second Affiliated Hospital, Zhejiang \\ University School of Medicine, Hangzhou, \\ Zhejiang Province, People's Republic of \\ China; ${ }^{2}$ Department of Radiology, \\ The Second Affiliated Hospital, Zhejiang \\ University School of Medicine, Hangzhou, \\ Zhejiang Province, People's Republic of \\ China; ${ }^{3}$ Department of Pathology, \\ The Second Affiliated Hospital, Zhejiang \\ University School of Medicine, Hangzhou, \\ Zhejiang Province, People's Republic of \\ China; ${ }^{4}$ Department of Surgical \\ Oncology, The Second Affiliated Hospital, \\ Zhejiang University School of Medicine, \\ Hangzhou, Zhejiang Province, People's \\ Republic of China
}

\begin{abstract}
Aggressive breast fibromatosis (referred to as a desmoid tumor) is a rare, locally invasive, non-metastasizing tumor with high recurrence rate. The therapeutic modalities range from surgery and radiotherapy to medical treatments. However, the optimal treatment is controversial, especially in a situation of repeated recurrence. Here, we present a case of a patient with aggressive breast fibromatosis with multiple recurrence after surgeries, who underwent high intensity focused ultrasound (HIFU) treatment effectively without side effects. To our knowledge, this is the first reported case of HIFU treatment in aggressive breast fibromatosis, which indicates that HIFU might be a novel, promising modality for this rare disease.
\end{abstract}

Keywords: aggressive breast fibromatosis, breast desmoid tumor, high intensity focused ultrasound, recurrence, treatment

\section{Introduction}

Aggressive fibromatosis, also referred to as desmoid tumor, is an unusual, locally invasive, non-metastasizing tumor, with high rates of local recurrence. ${ }^{1}$ It arises from fibroblasts or myofibroblasts and occurs more frequently in the abdominal wall and extremities but much rarely in the breast (only $0.2 \%$ of all breast neoplasms). ${ }^{2,3}$ There is scarce convictive evidence to support any treatment. ${ }^{4}$ Complete surgical resection might be the primary approach, however a front-line "watch-and-wait" policy has been recommended in recent years. For multiple recurrent cases, radiotherapy might be an option but side effects such as skin toxicity, tissue fibrosis, and radiation-induced neoplasms can appear. ${ }^{5}$ Systemic therapies such as cytotoxic chemotherapy, hormonal agents, nonsteroidal anti-inflammatory drugs (NSAIDS), and targeted therapies might be an option, but with limited efficacy. ${ }^{4}$

High intensity focused ultrasound (HIFU) is a novel, non-invasive ablation treatment which can cause thermal coagulation necrosis of the target lesion. Many studies of HIFU treatment have shown that it is a successful alternative modality in therapy for various malignant or benign tumors ${ }^{6-9}$ involving intraor extra-abdominal aggressive fibromatosis, but not the breast. ${ }^{10-13}$ Here, we report a case of aggressive breast fibromatosis with multiple recurrence after surgeries, treated with HIFU. The tumor showed remarkable regression. To our knowledge, this is the first reported case of HIFU treatment for this rare tumor located in the breast. 


\section{Case report}

A 26-year-old female with no previous medical history was admitted in June 2018 to the Department of Surgical Oncology, Second Affiliated Hospital of Zhejiang University, School of Medicine, for a repeatedly recurring right breast lump following two lumpectomies. Two years ago, she presented to a local hospital with a palpable right breast mass. Ultrasound showed an irregular, hypoechoic mass with obscure boundary ( $3 \mathrm{~cm}$ in diameter) in the right breast. She underwent her first lumpectomy in July 2016 at a local hospital. Histopathology showed a mass with irregular infiltration into adjacent adipose tissue (Figure 1A). High power evaluation showed poorly circumscribed spindle cells arranged in parallel and partially in wavy configuration around the mammary duct, with eosinophilic, pale cytoplasm, sparse nucleus chromatin, and unobvious nucleoli (Figure 1B and C). An immune-histochemical stain for $\beta$-catenin showed nuclear positivity (Figure 1D). There was no further radiotherapy or chemotherapy after the surgery. In November 2017, a $3.0 \times 3.0 \mathrm{~cm}$ breast mass in the area of the previous surgery showed up (Figure 1). She underwent a second lumpectomy. The pathology result was the same as last time. Likewise, no further therapeutic approaches were carried out. Six months later, a mass in the same region of the breast was palpated again. This time, she presented to our department.

Physical examination of the right breast revealed a firm, fixed $2 \mathrm{~cm}$ mass at the 8 o'clock position, beside the retracted nipple. On magnetic resonance imaging (MRI), a well-defined mass was observed at the rear of right breast nipple which showed homogeneous strengthening on enhanced magnetic resonance imaging (Figure 2A). The kinetic analysis demonstrated a plateau enhancement pattern. All these findings pointed to the recurrence of aggressive breast fibromatosis.

After obtaining informed consent, HIFU treatment was performed. She underwent two HIFU ablations on

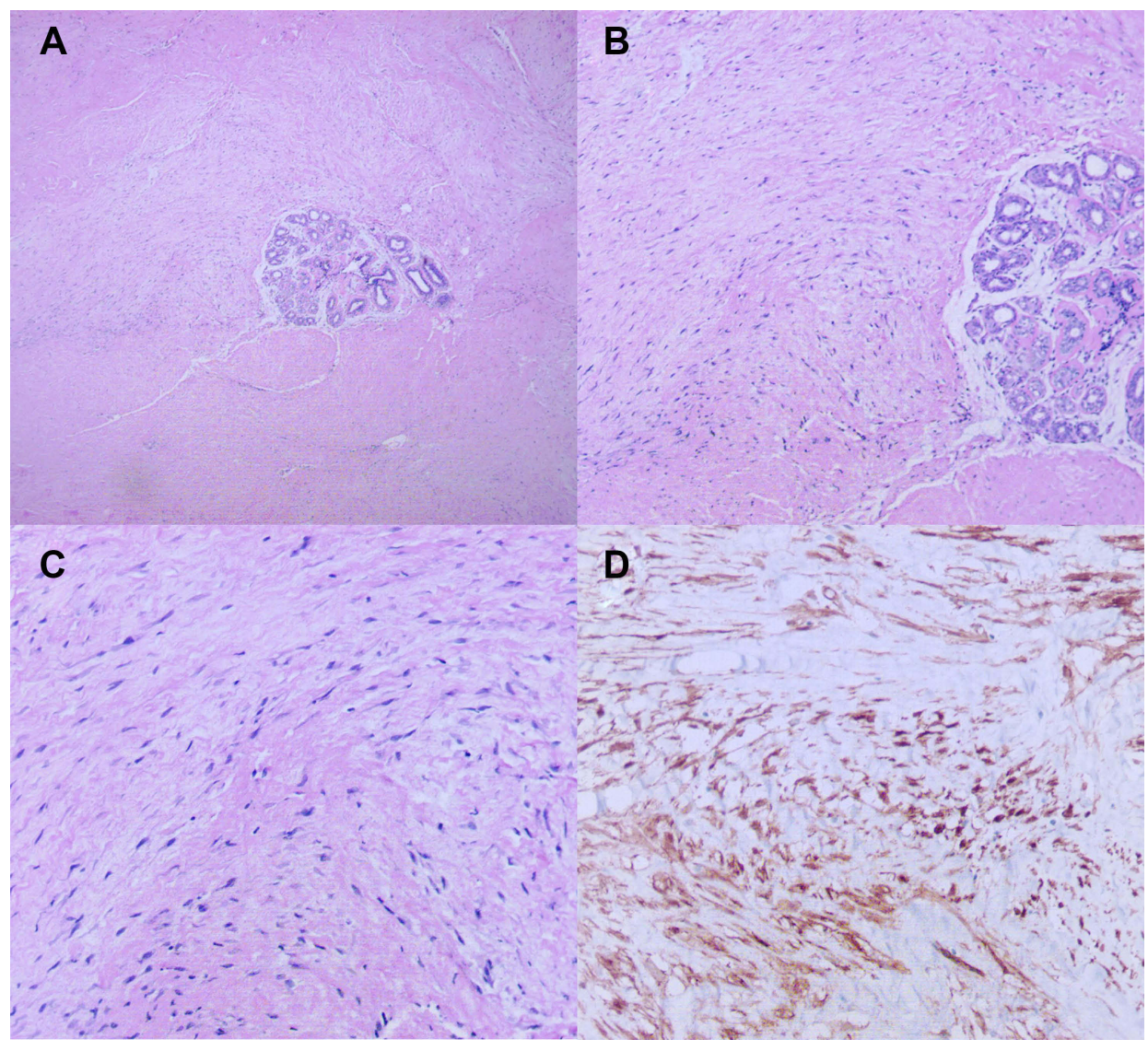

Figure I H\&E staining and immunohistochemical staining of specimens in the case. (A) The mass was irregular and infiltrated the adjacent adipose tissue (magnification, $\mathrm{x} 40$ ). (B) the spindle cells were arranged in parallel and partially in a wavy configuration around the mammary duct (magnification, $x \mid 00)$. (C) The spindle cells were poorly circumscribed with eosinophilic, pale cytoplasm, sparse nucleus chromatin, and unobvious nucleoli (magnification, $\times 200$ ). (D) Immunohistochemical stain for $\boldsymbol{\beta}$-catenin showed nuclear positivity. 


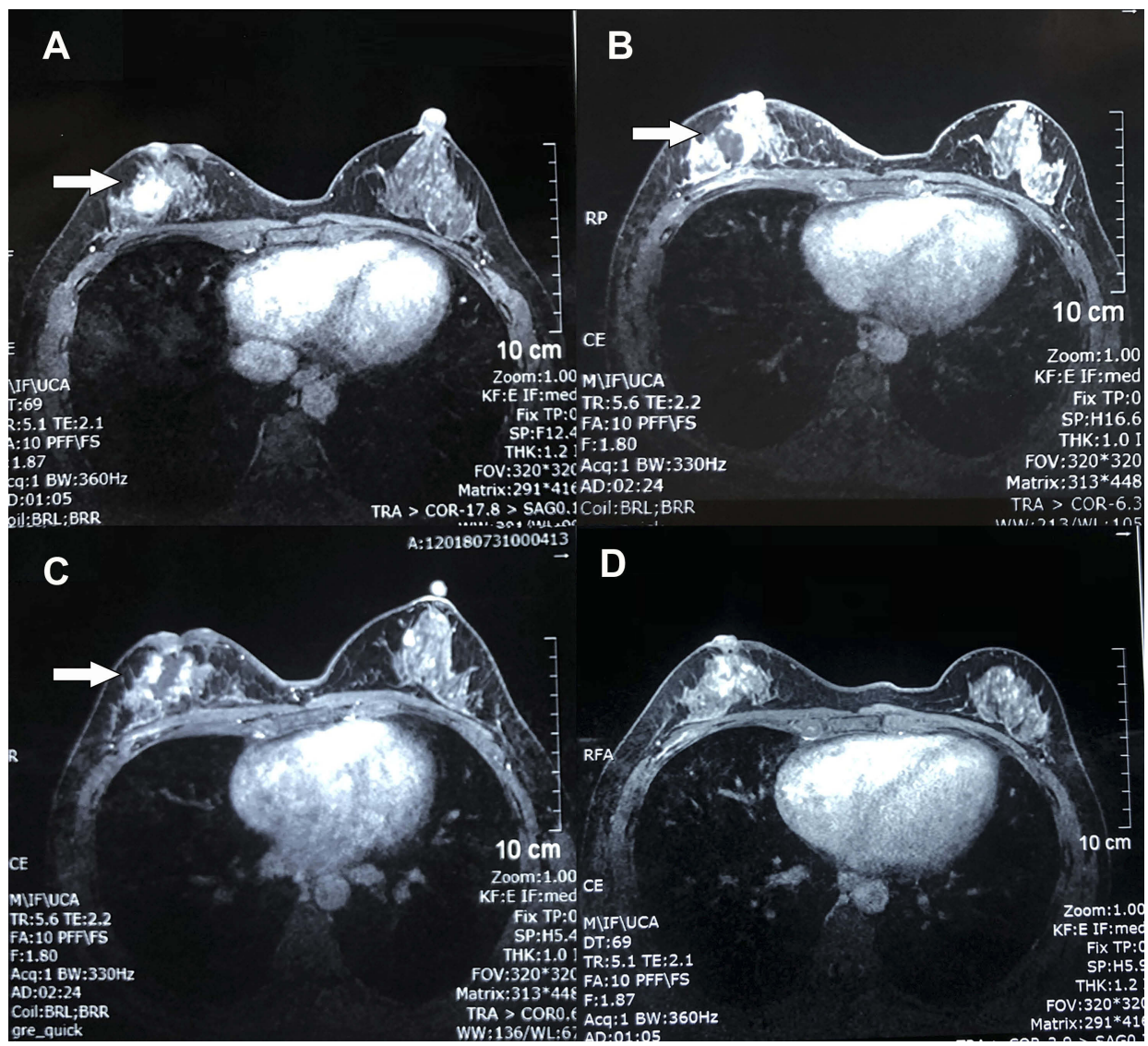

Figure 2 Contrast enhanced magnetic resonance imaging of the breast. (A) Before treatment, a well-defined mass (arrow) was observed at the rear of right breast nipple which showed homogeneous strengthening on enhanced magnetic resonance imaging. (B) Five days after the first high intensity focused ultrasound HIFU ablation, a noncontrast enhancement region (arrow) showed up in the previous mass area. (C) Five days after the second treatment, the non-contrast enhancement region was enlarged with irregular boundary and garland-like peripheral enhancement (arrow). (D) Three months after the last treatment, the mass was well ablated and only demonstrated a patchy, heterogeneous architectural distortion region. The nipple retraction was improved.

June 6th and July 25th, 2018, respectively. The HIFU procedure was performed with an FEPBY02 HIFU system (Yuande Biomedical Engineering Co. Ltd, Beijing, People's Republic of China) as described by $\mathrm{Hu}$ et al. ${ }^{14}$ The ablation effect was measured by contrast enhanced ultrasonography during HIFU treatment. The ultrasound images before and 48 minutes after treatment are shown in Figure 3. The HIFU parameters were as follows: ultrasonic power 280-300 W; emission time of T1 $200 \mathrm{~ms}$; interval time of T2 $200 \mathrm{~ms}$; total number of shots at each

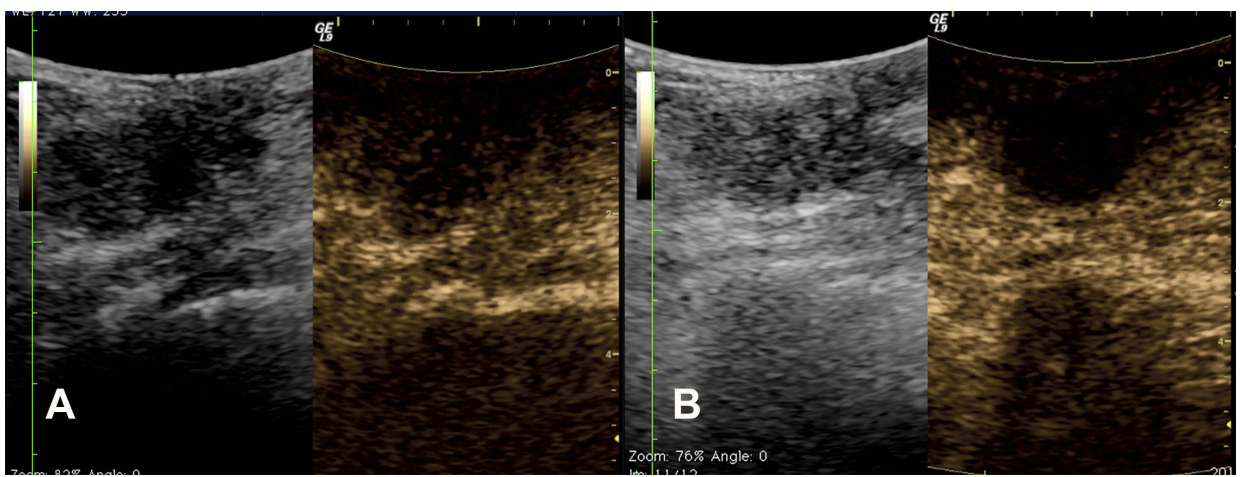

Figure 3 The contrast enhanced ultrasonography images before (A) and 48 minutes after treatment (B). (A) The left image shows a $3.0 \mathrm{~cm}$ irregular, hypoechoic mass with obscure boundary at right breast, located beside the nipple. The right image shows the blood flow signal inside the mass 52 seconds after contrast injection. (B) The left image shows the echo of mass enhanced; the right image shows non-contrast enhancement in the mass because of the vessel occlusion caused by high intensity focused ultrasound ablation. 
therapeutic point: 25 times (lasted 10 seconds); a spacing of $1 \mathrm{~mm}$ between adjacent treatment slices. Three therapeutic points composed one unit with 30 seconds interval between each unit. The whole therapeutic process was divided into three sections consisting of five therapeutic units for each. The total emission lasted 3 minutes and 45 seconds and the whole therapeutic schedule lasted 30 minutes. Five days after the first treatment, MRI showed non-contrast enhancement region in the mass, which indicated there was no blood flow (Figure 2B). Five days after the second treatment, the non-contrast enhancement region on previous MRI was enlarged with irregular boundary and garland-like peripheral enhancement (Figure 2C). These signs demonstrated that tumor necrosis developed. We suspected the peripheral enhancement might be the granulation tissue restoration. Three months after the last treatment, the nipple retraction improved in appearance. The breast MRI demonstrated a patchy, heterogeneous architectural distortion, and non-contrast enhancement region at the rear of right breast nipple (Figure 2D). The necrotic region had been absorbed. These signs indicated that the lesion was completely ablated and restored with granulation tissue. The patient was advised to undergo regular follow-up with ultrasound at 3-month intervals. Up to now, there has been no sign of recurrence. Written informed consent was obtained from the patient to have the case details and any accompanying images published. The case details were approved for publication by the Second Affiliated Hospital, Zhejiang University School of Medicine.

\section{Discussion}

Aggressive fibromatosis, also referred to as desmoid tumor, is a rare clonal proliferation of fibroblasts and myofibroblasts. It occurs more frequently in the abdominal wall and extremities but rarely in the breast (only $0.2 \%$ of all breast tumors), ${ }^{3}$ and resembles breast carcinomas clinically and radiologically. This entity is thought to lack metastatic potential, but is locally aggressive and prone to recurrence. ${ }^{2}$ The real etiology of breast fibromatosis is unknown. It has been reported that it might be correlated with incidental or surgical trauma and breast implants, and is associated with hereditary diseases such as Gardner syndrome and familial adenomatous polyposis. ${ }^{15}$

Up until now, there has been no standard therapeutic strategy for aggressive breast fibromatosis. Complete surgical resection seems to be the generally agreed upon primary treatment. However, $27 \%$ of patients still experience recurrence after surgery despite clear margins. ${ }^{16}$ Further surgery may cause significant morbidity, for surgery itself as an iatrogenic trauma and one of the causes of fibromatosis. It has been reported that some patients undergo repeated surgeries, ${ }^{17}$ just like this case, which badly destroys the appearance of the breast and patients' quality of life. Besides aggressive treatment, active observation has been another strategy recommended recently. A $28 \%$ spontaneous regression rate has been reported in an observation group of patients with extra-abdominal desmoid fibromatosis. ${ }^{18}$ The National Comprehensive Cancer Network (NCCN) 2018 guidelines for soft tissue sarcoma recommend observation as an option for selected patients in non-life threatening situations. ${ }^{19}$ However, the criterion of "selected patients" is still perplexing. There are no significant prognostic factors to identify these patients. ${ }^{20}$ Once the disease has progressed, more aggressive surgical resection would be performed. Radiotherapy is another treatment option, especially for unresectable lesions. Studies have shown that it increases the local control compared to surgery alone. ${ }^{21}$ Nevertheless, the efficacy data for aggressive breast fibromatosis are scarce. The optimal regimes, doses, and durations are still controversial. Although the acute side effects have been tolerable, the late effects such as second primary tumor in the relatively young population, deserve more attention ${ }^{22,23}$ as the majority of patients will live for many decades. Systemic therapies for aggressive breast fibromatosis include cytotoxic chemotherapy, hormonal manipulations, NSAIDS, and biological therapies such as imatinib and sorafenib. ${ }^{4,24}$ However, the majority of data were derived from small cohorts evaluating extra-mammary fibromatosis. Due to the limited evidence, the 2018 NCCN guidelines recommend systemic therapy as an option for advanced or unresectable desmoid tumors. ${ }^{19}$

HIFU is a novel, non-invasive modality for thermal ablation. It focuses ultrasound beams on the target lesion precisely and causes thermal coagulation necrosis without damaging adjacent tissue. ${ }^{6,25}$ As a non-invasive technique, there is no insertion applicators, which avoids iatrogenic trauma as a possible etiological factor of aggressive fibromatosis. ${ }^{15}$ Besides, the acoustic energy only accumulates in the focal lesion but not in intervening tissue, ${ }^{25}$ which reduces the damage to adjacent tissue, meaning HIFU has less side effects and is well-tolerated. In People's Republic of China, it has been approved by China Food and Drug Administration for the treatment of locally advanced or metastatic pancreatic cancer because of its excellent local control rate and benefit in survival. 
In the past decade, HIFU has been well-documented as a successful alternative modality in various malignant or benign tumors, such as liver tumor, ${ }^{8}$ bone malignancy, ${ }^{9}$ prostate cancer, ${ }^{26}$ and thyroid nodules. ${ }^{7}$ In prostate cancer, for instance, the efficacy of HIFU in locally confined disease is now comparable to those of radiotherapy and radical prostatectomy. ${ }^{26}$ The complications were more less in HIFU group. ${ }^{27}$ However, reports of the efficacy in aggressive fibromatosis are scarce. Most of them were focused on intraor extra-abdominal locations without involvement of the breast. ${ }^{10-13}$ Ghanouni $^{10}$ revised 15 patients with extraabdominal aggressive fibromatosis who underwent HIFU. The mean tumor volume decreased in $63 \%$ with significant improvement in pain. Besides the promising efficacy, the side effects were tolerable. Low-grade fever, thrombocytopenia, skin burns, and nerve injury were observed, which resolved quickly. Unlike radiotherapy, there is no accumulated dose limitation for HIFU which means it can be performed repeatedly. Although the current data are based on small samples, the potential of HIFU in treatment of this rare, locally aggressive tumor is promising. Here, we presented a case of aggressive breast fibromatosis with multiple recurrence after surgeries. The patient was a young, beautiful lady troubled by the appearance of nipple retraction. Five days after the first treatment, the MRI showed the blood supply of the lesion was cut off significantly. Then the necrosis started, followed by granulation tissue restoration. The lesion was completely ablated after two HIFU treatments without any side effects and the nipple retraction improved significantly, which meant a lot to her. The lesion has been stable and without perfusion on MRI up until now. To our knowledge, this is the first reported case of treatment of aggressive breast fibromatosis with HIFU, which indicates HIFU might be a novel, promising modality for this rare disease.

\section{Conclusion}

In conclusion, aggressive breast fibromatosis is a rare, locally aggressive tumor with high incidence of recurrence. Management of breast fibromatosis remains controversial. HIFU ablation avoids the trauma caused by surgery, side effects caused by radiation and chemotherapy, and shows remarkable and durable efficacy in local control. It might be a promising novel modality for treatment of recurrent aggressive breast fibromatosis. Further studies are expected.

\section{Informed consent}

Written informed consent was obtained from the patient to have the case details and any accompanying images published. The case details were approved for publication by the Second Affiliated Hospital, Zhejiang University School of Medicine.

\section{Disclosure}

The authors report no conflicts of interest in this work.

\section{References}

1. Otero S, Moskovic EC, Strauss DC, et al. Desmoid-type fibromatosis. Clin Radiol. 2015;70(9):1038-1045. doi:10.1016/j.crad.2015.04.015

2. Glazebrook KN, Reynolds CA. Mammary fibromatosis. AJR Am J Roentgenol. 2009;193(3):856-860. doi:10.2214/AJR.08.1892

3. Schwarz GS, Drotman M, Rosenblatt R, Milner L, Shamonki J, Osborne MP. Fibromatosis of the breast: case report and current concepts in the management of an uncommon lesion. Breast J. 2006;12(1):66-71. doi:10.1111/j.1075-122X.2006.00187.x

4. Martinez Trufero J, Pajares Bernad I, Torres Ramon I, Hernando Cubero J, Pazo Cid R. Desmoid-type fibromatosis: who, when, and how to treat. Curr Treat Options Oncol. 2017;18(5):29. doi:10.1007/ s11864-017-0474-0

5. Scheer L, Lodi M, Moliere S, Kurtz JE, Mathelin C. Medical treatment of mammary desmoid-type fibromatosis: which benefit? World J Surg Oncol. 2017;15(1):86. doi:10.1186/s12957-017-1148-x

6. Rombouts SJ, Vogel JA, van Santvoort HC, et al. Systematic review of innovative ablative therapies for the treatment of locally advanced pancreatic cancer. Br J Surg. 2015;102(3):182-193. doi:10.1002/ bjs. 9716

7. Lang BH, Woo YC, Chiu KW. Role of second high-intensity focused ultrasound (HIFU) treatment for unsatisfactory benign thyroid nodules after first treatment. Eur Radiol. 2019;29(3):1469-1478.

8. Aubry JF, Pauly KB, Moonen C, et al. The road to clinical use of high-intensity focused ultrasound for liver cancer: technical and clinical consensus. J Ther Ultrasound. 2013;1:13. doi:10.1186/ 2050-5736-1-13

9. Chen W, Zhu H, Zhang L, et al. Primary bone malignancy: effective treatment with high-intensity focused ultrasound ablation. Radiology. 2010;255(3):967-978. doi:10.1148/radiol.10090374

10. Ghanouni P, Dobrotwir A, Bazzocchi A, et al. Magnetic resonance-guided focused ultrasound treatment of extra-abdominal desmoid tumors: a retrospective multicenter study. Eur Radiol. 2017;27(2):732-740. doi:10.1007/s00330-016-4376-5

11. Shi Y, Huang Y, Zhou M, Ying X, Hu X. High-intensity focused ultrasound treatment for intra-abdominal desmoid tumors: a report of four cases. J Med Ultrason (2001). 2016;43(2):279-284. doi:10.1007/s10396-015-0682-9

12. Wang Y, Wang W, Tang J. Ultrasound-guided high intensity focused ultrasound treatment for extra-abdominal desmoid tumours: preliminary results. Int J Hyperthermia. 2011;27(7):648-653. doi:10.3109/ 02656736.2011.597047

13. Zhao WP, Han ZY, Zhang J, et al. Early experience: high-intensity focused ultrasound treatment for intra-abdominal aggressive fibromatosis of failure in surgery. Br $J$ Radiol. 2016;89(1062):20151026. doi:10.1259/bjr.20151026

14. Hu X, Cai H, Zhou M, et al. New clinical application of high-intensity focused ultrasound: local control of synovial sarcoma. World J Surg Oncol. 2013;11:265. doi:10.1186/1477-7819-11-265

15. Grimaldi MC, Trentin C, Lo Gullo R, Cassano E. Fibromatosis of the breast mimicking cancer: a case report. Radiol Case Rep. 2018;13 (1):1-5. doi:10.1016/j.radcr.2017.09.011

16. Ballo MT, Zagars GK, Pollack A, Pisters PW, Pollack RA. Desmoid tumor: prognostic factors and outcome after surgery, radiation therapy, or combined surgery and radiation therapy. J Clin Oncol. 1999;17(1):158-167. doi:10.1200/JCO.1999.17.1.158 
17. Povoski SP, Marsh WL Jr., Spigos DG, Abbas AE, Buchele BA. Management of a patient with multiple recurrences of fibromatosis (desmoid tumor) of the breast involving the chest wall musculature. World J Surg Oncol. 2006;4:32. doi:10.1186/14777819-4-32

18. Bonvalot S, Ternes N, Fiore M, et al. Spontaneous regression of primary abdominal wall desmoid tumors: more common than previously thought. Ann Surg Oncol. 2013;20(13):4096-4102. doi:10.1245/s10434-013-3197-x

19. NCCN clinical practice guideline for sarcoma 2018 Version 2. Available from:https://www.nccn.org/professionals/physician_gls/ pdf/sarcoma.pdf. Accessed June 10, 2019.

20. Kasper B, Baumgarten C, Garcia J, et al. An update on the management of sporadic desmoid-type fibromatosis: a European consensus initiative between Sarcoma PAtients EuroNet (SPAEN) and European Organization for research and treatment of cancer (EORTC)/Soft Tissue and Bone Sarcoma Group (STBSG). Ann Oncol. 2017;28(10):2399-2408. doi:10.1093/annonc/mdx323

21. Janssen ML, van Broekhoven DL, Cates JM, et al. Meta-analysis of the influence of surgical margin and adjuvant radiotherapy on local recurrence after resection of sporadic desmoid-type fibromatosis. $\mathrm{Br} \quad J$ Surg. 2017;104(4):347-357. doi:10.1002/ bjs. 10477
22. Keus RB, Nout RA, Blay JY, et al. Results of a phase II pilot study of moderate dose radiotherapy for inoperable desmoid-type fibromatosis-an EORTC STBSG and ROG study (EORTC 62991-22998). Ann Oncol. 2013;24(10):2672-2676. doi:10.1093/annonc/mdt254

23. Bates JE, Morris CG, Iovino NM, et al. Radiation therapy for aggressive fibromatosis: the association between local control and age. Int $J$ Radiat Oncol Biol Phys. 2018;100(4):997-1003. doi:10.1016/j.ijrobp.2017.12.259

24. Plaza MJ, Yepes M. Breast fibromatosis response to tamoxifen: dynamic MRI findings and review of the current treatment options. J Radiol Case Rep. 2012;6(3):16-23. doi:10.3941/jrcr.v6i3.897

25. Zhou Y. High-intensity focused ultrasound treatment for advanced pancreatic cancer. Gastroenterol Res Pract. 2014;2014:205325. doi: $10.1155 / 2014 / 205325$

26. Chaussy CG, Thuroff S. High-intensity focused ultrasound for the treatment of prostate cancer: a review. J Endourol. 2017;31(S1):S30 S37. doi:10.1089/end.2016.0548

27. Devos B, Al Hajj Obeid W, Andrianne C, et al. Salvage high-intensity focused ultrasound versus salvage radical prostatectomy for radiation-recurrent prostate cancer: a comparative study of oncological, functional, and toxicity outcomes. World J Urol. 2019. doi:10.1007/s00345-019-02640-x.

\section{Publish your work in this journal}

OncoTargets and Therapy is an international, peer-reviewed, open access journal focusing on the pathological basis of all cancers, potential targets for therapy and treatment protocols employed to improve the management of cancer patients. The journal also focuses on the impact of management programs and new therapeutic agents and protocols on patient perspectives such as quality of life, adherence and satisfaction. The manuscript management system is completely online and includes a very quick and fair peer-review system, which is all easy to use. Visit http://www.dovepress.com/ testimonials.php to read real quotes from published authors. 